\title{
Overdenture retida por implante único: Uma alternativa para a reabilitação oral de idosos
}

\author{
Kassia Hauck*, Thamiris Nogueira Sacker**, Valquíria de Jesus Freitas ${ }^{\star \star *}$,

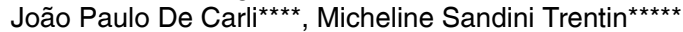

\section{Resumo}

Com o aumento da expectativa de vida, há o aumento da procura dos pacientes edêntulos por uma forma reabilitadora que lhes proporcione maior eficiência mastigatória. As sobredentaduras sobre implantes utilizando sistemas de encaixe representam modalidade protética mucossuportada e implantorretida que possibilita segurança, estabilidade e estética, menor custo e facilidade para higienização. Internacionalmente, dois implantes são considerados adequados para manter uma overdenture nas mandíbulas desdentadas. No entanto, a instalação de dois implantes pode representar um procedimento invasivo e oneroso. Este trabalho objetiva demonstrar que a inserção de um único implante na porção central da mandíbula pode estabilizar a prótese total e melhorar a qualidade de vida e a função mastigatória da saúde bucal. O estudo trata de um relato de caso de um paciente masculino, de 65 anos de idade, que relatava falta de estabilidade da prótese total inferior convencional, confeccionada há vários anos. O tratamento proposto foi a instalação de implante único em sínfise mandibular e posterior confecção de novas próteses totais. Uma fixação com pilar tipo o'ring e matriz ativada por parafuso foi usada para manter a sobredentadura inferior. Os resultados clínicos mostraram-se promissores, sendo que o paciente relata melhora na mastigação, estabilidade e função da prótese retida por implante único. No entanto, para que esse método possa ser recomendado para aplicação clínica geral, são necessários resultados de estudos clínicos longitudinais.

Palavras-chave: Sobredentadura. Implantes. Implante único. Idosos.

* Especialista em Implantodontia e Cirurgia e Traumatologia Bucomaxilofaciais, aluna do curso de Mestrado em Clínica Odontológica da Faculdade de Odontologia da UPF.

** Mestre em Odontologia pela ULBRA/RS, aluna do curso de Doutorado em Clínica Odontológica da Faculdade de Odontologia da UPF.

*** Acadêmica do Curso de Odontologia da Faculdade de Odontologia da UPF.

**** Especialista em Prótese Dentária, Mestre e Doutor em Estomatologia; Professor dos cursos de Graduação e Pós-Graduação da Faculdade de Odontologia da UPF.

****** Especialista em Implantodontia, Mestre e Doutora em Periodontia; Professora dos cursos de Graduação e Pós-Graduação da Faculdade de Odontologia da UPF.

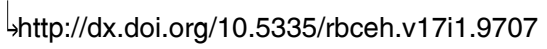




\section{Introdução}

A população brasileira manteve a tendência de envelhecimento dos últimos anos superando a marca dos 30,2 milhões de idosos em 2017. Em 2012, a população com 60 anos ou mais era de 25,4 milhões. Desde 2012 ocorreu um aumento de 4,8 milhões de novos idosos em cinco anos que corresponde a um crescimento de $18 \%$ desse grupo etário, que tem se tornado cada vez mais representativo no Brasil. As mulheres são maioria expressiva nesse grupo, com 16,9 milhões (56\% dos idosos), enquanto os homens idosos são 13,3 milhões (44\% do grupo) (IBGE, 2017).

Com relação à perda dentária, os dados da Pesquisa Nacional de Saúde Bucal evidenciaram que ocorreu em 25\% dos adultos, sendo que a média de dentes perdidos variou de 13,5 em 2002/2003 para 7,4 em 2010. Entretanto, mais da metade dos idosos tem edentulismo, tanto em 2010 quanto em 2002/2003 (NICO et al., 2016).

Inúmeras consequências sistêmicas e locais são percebidas nos indivíduos desdentados, dentre elas: comprometimento estético, mastigatório, nutritivo e na fonação, assim como redução da autoestima, podendo levar o paciente edêntulo (especialmente quando idoso) a uma situação de isolamento social e depressão (CARLSSON; OMAR, 2010; HUGO et al., 2007; MUSACCHIO et al., 2007).

A opção de reabilitação oral mais executada em desdentados totais é a prótese total mucossuportada, sendo a retenção e estabilidade fatores determinantes fundamentais para os resultados de sucesso. Embora a maior parte dos indivíduos reabilitados com próteses totais convencionais esteja satisfeita, uma parcela significativa apresenta reclamações quanto à estética, retenção e função, especialmente no que diz respeito à arcada inferior (CRITCHLOW; ELLIS, 2010; GROVER; VAIDYANATHAN; VEERAVALLI, 2014; HYLAND et al., 2009; SMITH; BAYSAN; FENLON, 2009).

Uma alternativa para melhorar a retenção, estabilidade e aspectos funcionais das próteses totais inferiores em pacientes com prognóstico desfavorável devido à morfologia do rebordo é a utilização de implantes osseointegráveis, seja para retenção de prótese total fixa ou para overdentures. Porém, a definição do número de implantes necessários para sustentar a overdenture mandibular em pacientes com atrofia mandibular severa ainda é tema controverso na literatura (CORDIOLI; MAJZOUB; CASTAGNA, 1997; KLEMETTI, 2008).

Com o aumento da expectativa de vida, há o aumento da procura dos pacientes edêntulos por uma forma reabilitadora que lhes proporcione maior eficiência mastigatória. As sobredentaduras sobre implantes utilizando sistemas de encaixe bola/o'ring e barra-clip representam modalidade protética mucossuportada e implantorretida que possibilita maior segurança, estabilidade e estética, além de apresentarem menor custo e facilidade para higienização (NOGUEIRA et al., 2018). 
Ensaio clínico randomizado mostrou que dois implantes na região mandibular anterior promovem significativa melhora na retenção e estabilidade das próteses totais inferiores. A melhoria desses parâmetros (semelhante a quando se utilizava um maior número de implantes), a sobrevida dos implantes, simplificação do tratamento e a menor exigência das condições de saúde ideais do paciente foram fatores preponderantes para o êxito no uso de overdentures inferiores retidas por dois implantes na região mentoniana (THOMASON et al., 2003).

O sucesso desta modalidade de tratamento, embora notável, é de alto custo para muitos indivíduos edêntulos. Um estudo de comparação de custos entre overdentures mandibulares retidas por 2 implantes e próteses totais inferiores convencionais mostrou que o custo da overdenture é 2,4 vezes o custo da prótese total convencional (TAKANASHI et al., 2004). É desejável, portanto, que os clínicos possam oferecer melhora funcional em relação às próteses inferiores numa adequada relação custo-benefício. Além disso, é desejável a redução no tempo total de procedimentos clínicos, técnicos e de manutenção para atingir os objetivos do tratamento.

A overdenture mandibular retida por um único implante localizado na sínfise mandibular foi proposta como alternativa de tratamento para pacientes idosos com experiência prévia de desconforto e dificuldades funcionais com as próteses totais convencionais (CORDIOLI; MAJZOUB; CASTAGNA, 1997). No entanto, poucos estudos abordam esta opção de tratamento, principalmente no que diz respeito a ensaios clínicos randomizados e amostras compostas por número considerável de indivíduos (KRONSTROM et al., 2010; LIDDELOW; HENRY, 2007). Estudos relatam que a ausência de retenção e de estabilidade da prótese mandibular é o principal motivo para a insatisfação das próteses dentárias e tem um impacto direto na qualidade de vida relacionada à saúde bucal (SCHUSTER et al., 2017).

O objetivo deste estudo é relatar um incomum caso clínico de overdenture retida por implante único instalado na região central da mandíbula de paciente idoso, utilizando-se o sistema de retenção por meio de pilar do tipo bola/o'ring com anel de borracha.

\section{Relato de caso clínico}

Paciente masculino, 65 anos de idade, leucoderma, com boa saúde geral, procurou atendimento odontológico por apresentar perda de estabilidade e retenção da sua prótese total convencional inferior. Tal prótese, juntamente com uma prótese total superior, era utilizada há mais de 10 anos. Foi proposta para o paciente a instalação de um implante único na região central da mandíbula, com posterior confecção de uma overdenture mucoimplantossuportada inferior $\mathrm{e}$ de uma nova prótese total convencional superior (Figura 1), conforme recomendações (ABOU-AYASH et al., 2020; CANALLATOS et al., 2020; ISMAEL et al., 2015; PATIL; SEOW, 2020). 
Figura 1: Situação clínica inicial do paciente. Nota-se a presença de rebordo residual inferior atrófico, o que dificulta a utilização da prótese total inferior convencional

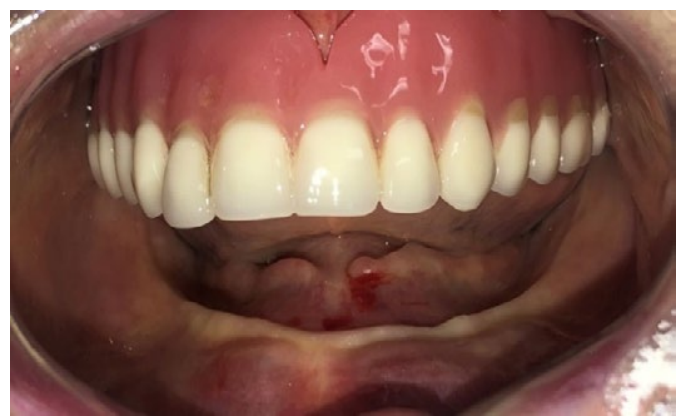

De posse de exames complementares laboratoriais com resultados satisfatórios e de radiografia panorâmica, procedeu-se à escolha do implante para o caso clínico.

Para tal, realizou-se planejamento reverso a partir da duplicação da prótese total antiga do paciente e obteve-se um guia cirúrgico para instalação do implante (Singular Implants ${ }^{\circledR}$, Parnamirim, $\mathrm{RN}$, Brasil) do tipo cone-morse (4.0 mm x $10 \mathrm{~mm}$ ), cuja plataforma foi posicionada $2 \mathrm{~mm}$ abaixo do nível ósseo mandibular (Figura 2).

O protocolo medicamentoso consistiu na utilização de antibiótico (amoxicilina $500 \mathrm{mg}$, dois comprimidos 1 hora antes do procedimento e após um comprimido de 8/8 horas por sete dias); antinflamatório (nimesulida $100 \mathrm{mg}$, um comprimido de 12/12 horas por três dias) e analgésico (paracetamol $750 \mathrm{mg}$, um comprimido de 8/8 horas durante três dias), além de dois bochechos diários com solução de clorexidine $0,12 \%$ sem álcool por 10 dias consecutivos, iniciando no dia anterior à cirurgia de instalação do implante.

Figura 2: A) Retalho na crista do rebordo inferior e incisões relaxantes realizadas; B) Perfuração do tecido ósseo para instalação do implante; C) Implante instalado na região de sínfise mandibular
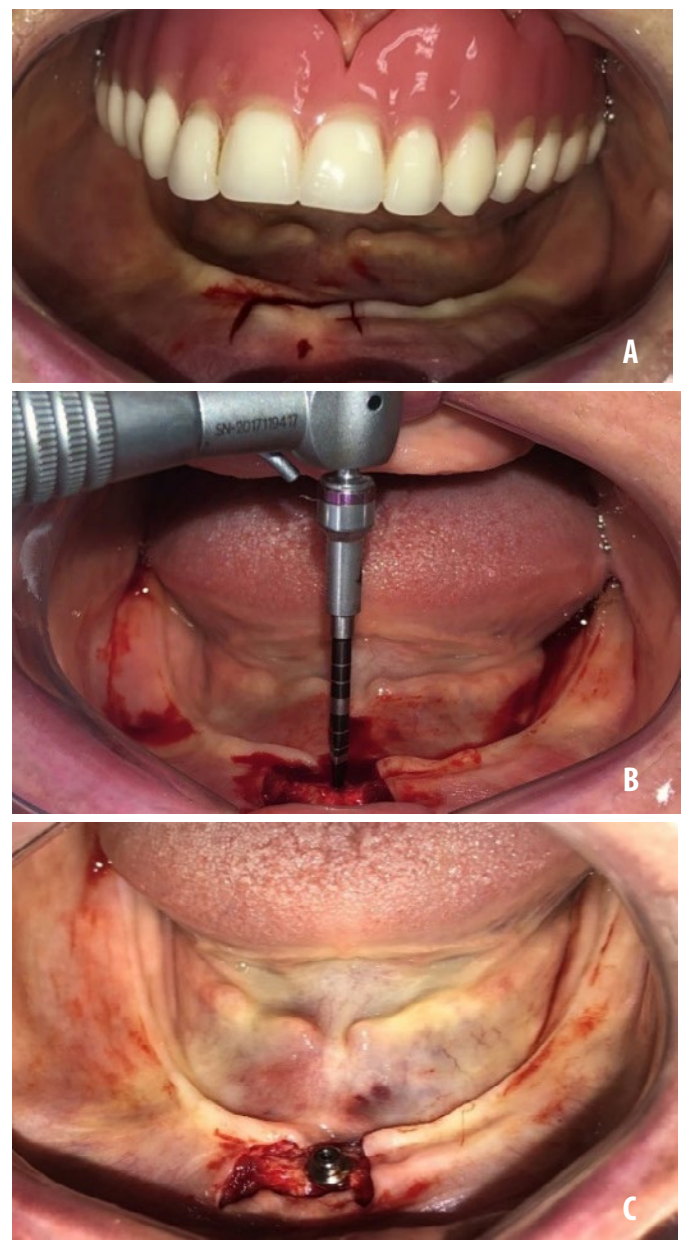

Após a cirurgia aguardou-se o período de cicatrização de quatro meses e procedeu-se ao segundo tempo cirúrgico (Figuras 3 e 4). Dez dias após, foi insta- 
lado um pilar protético do tipo bola/o'ring com altura de cinta metálica de $3 \mathrm{~mm}$, emergindo $1 \mathrm{~mm}$ da margem gengival. O torque do pilar protético foi de $32 \mathrm{~N}$, conforme recomendação do fabricante, tendo sido dado um retorque após 10 minutos de acordo com estudos (CORDIOLI; MAJZOUB; CASTAGNA,1997; KRONSTROM et al., 2010; TAVAKOLIZADEH et al. 2015).

Figura 3 - Radiografia panorâmica obtida previamente ao segundo tempo cirúrgico, mostrando condição satisfatória de osseointegração do mesmo

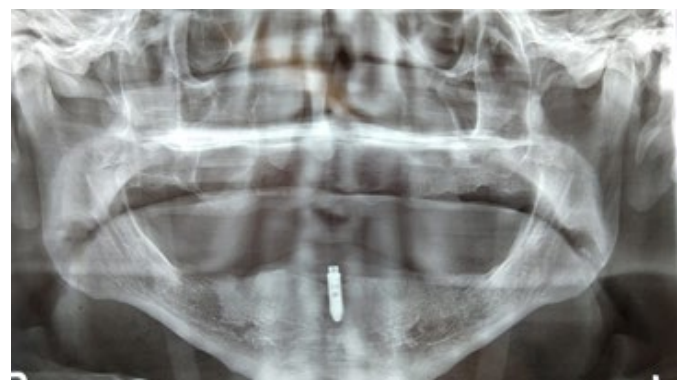

Figura 4: Cicatrizador instalado durante 10 dias na cavidade bucal previamente ao procedimento de moldagem/ transferência do implante

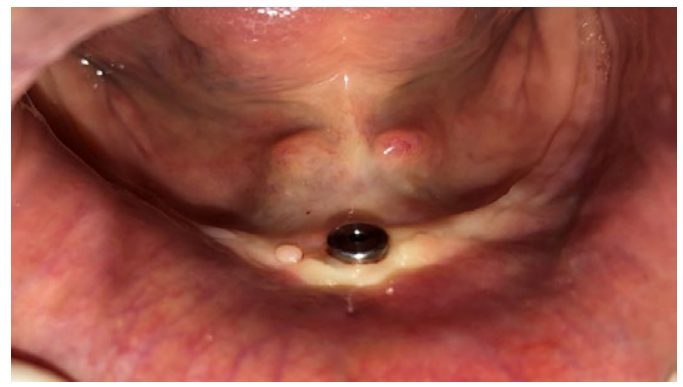

Concomitante ao segundo tempo cirúrgico iniciou-se a confecção das novas próteses totais do paciente. As moldagens foram confeccionadas com silicone de condensação (Zetaplus ${ }^{\circledR}$, Labordental Ltda, São Paulo, SP, Brasil) no qual foram realizadas retenções e moldagem corretiva com hidrocolóide irreversível (Jeltrate $^{\circledR}$, Dentsply Indústria e Comércio Ltda., Petrópolis, Rio de Janeiro, RJ, Brasil) em moldeiras de estoque perfuradas. Após a confecção dos modelos de gesso, foi realizada a confecção de moldeiras individuais em resina acrílica autopolimerizável incolor (Clássico ${ }^{\circledR}$, Artigos Odontológicos Clássico Ltda., Campo Limpo Paulista, SP, Brasil) para posterior vedamento periférico utilizando godiva em bastão (Exata ${ }^{\circledR}$, DFL Indústria e Comércio S. A., Rio de Janeiro, RJ, Brasil) e moldagem funcional, realizada com Poliéter (Impregum ${ }^{\circledR}$, 3M Produtos Odontológicos, Sumaré, SP, Brasil).

A captura da cápsula na prótese inferior foi feita posicionando a porção metálica no pilar o`ring, e realizando um alívio na porção interna da prótese na região correspondente ao implante. Após foi introduzida resina acrílica autopolimerizável no orifício e as próteses foram inseridas em boca até a polimerização total da resina, em posição de relação cêntrica conforme autores KRONSTROM et al., 2010; TAVAKOLIZADEH et al. 2015 (Figura 5). 
Figura 5: A) Pilar o'ring instalado sobre o implante; B) Cápsula com anel de borracha capturada com resina acrílica na porção interna da prótese inferior
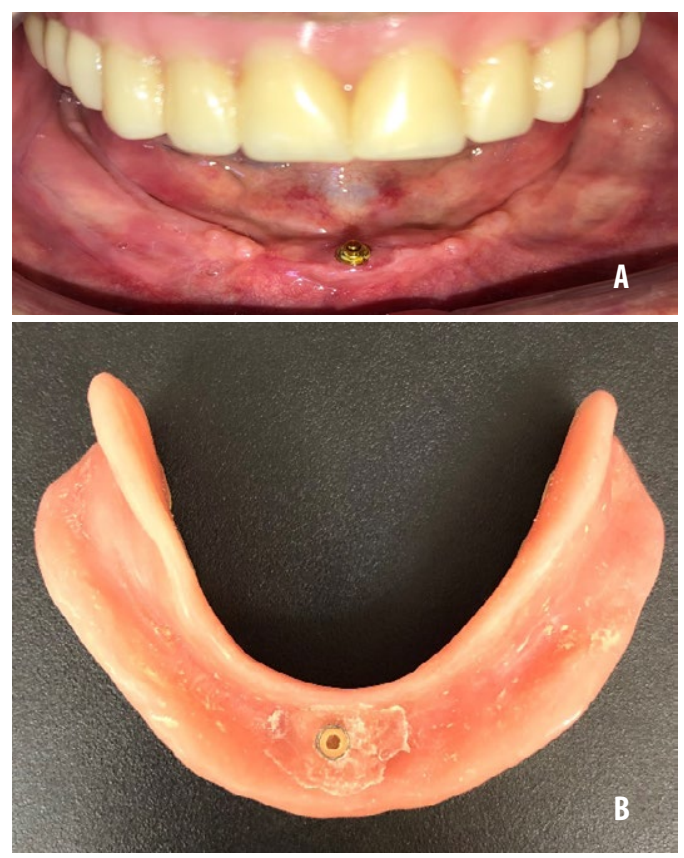

Em seguida, foi realizado o acabamento e polimento na região da cápsula para evitar ranhuras na superfície interna da prótese, além do ajuste oclusal. $\mathrm{O}$ paciente foi instruído em relação à colocação e retirada das próteses, e quanto a higienização (Figura 6).
Figura 6: Próteses superior e inferior instaladas na cavidade bucal. Pode-se notar engrenamento oclusal e aspecto estético satisfatório

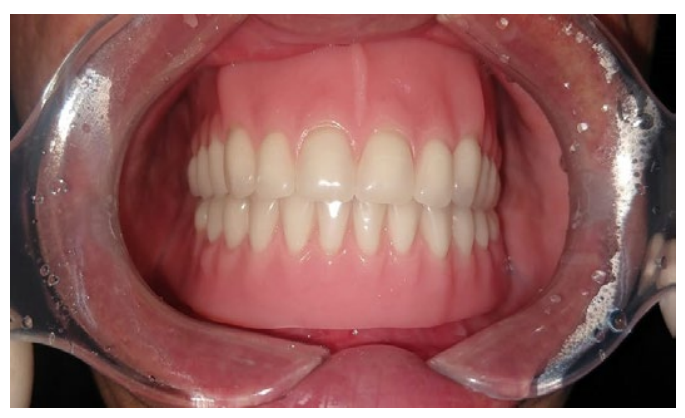

O paciente permitiu a publicação do presente trabalho por meio da assinatura de um Termo de Consentimento Livre e Esclarecido. Além disso, o presente trabalho foi aprovado pelo Comitê de Ética em Pesquisa da Universidade de Passo Fundo (Parecer oㅜ 2.572.556).

\section{Discussão}

De acordo com Mahookar et al. (2016), o implante de sistema único foi aprovado com uma excelente relação custo-benefício e tratamento clínico de sucesso.

Os indivíduos que têm dificuldades com suas próteses geralmente se queixam de dor, desconforto, coordenação oral deficiente e funcionamento oral limitado, principalmente por baixa retenção, estabilidade e condição desfavorável dos tecidos de suporte associados à prótese inferior. Na maioria dos casos, recomenda-se o uso de implantes dentários para reter uma prótese inferior e assegurar um prognóstico mais favorável 
para os pacientes com arcos edêntulos difíceis de reabilitar com prótese total inferior convencional (ABOU-AYASH, 2020; NOGUEIRA; ESFANDIARI; LELES, 2016). Em nosso relato o idoso em questão também procurou atendimento queixando-se da perda de estabilidade e retenção da sua prótese total convencional inferior, confeccionada há mais de dez anos, frente ao que se sugeriu a reabilitação com overdenture inferior retida por implante único.

A sobredentadura retida por implante é uma opção para um paciente completamente edêntulo. $\mathrm{O}$ uso de um número mínimo de implantes adequados para o suporte e a retenção de próteses pode trazer uma melhoria na qualidade de vida e menores custos para os pacientes (BRYANT; WALTON; MACENTEE, 2015; LAHOTI; PATHRABE; GADE, 2016; NISCHAL; CHOWDHARY, 2016 PASSIA; WOLFART; KERN, 2015). No presente relato de caso, visando minimizar custos e tendo-se em vista o padrão oclusal com ausência de parafunção, optou-se pela instalação de um único implante em região de sínfise mandibular. Tal modalidade de tratamento, apesar de ter sido descrita há vários anos (CORDIOLI; MAJZOUB; CASTAGNA, 1997), ainda é pouco abordada na literatura científica. Busca-se assim, com o presente estudo, realçar um tratamento pouco invasivo e menos oneroso, que melhora significativamente a qualidade de vida dos pacientes edêntulos, especialmente os idosos. (ABOU-AYASH, 2020; LAHOTI; PATHRABE; GADE, 2016; NISCHAL; CHOWDHARY, 2016;
NOGUEIRA; ESFANDIARI; LELES, 2016, PATIL; SEOW, 2020).

O prognóstico de uma overdenture depende de dois importantes fatores: retenção e distribuição de tensão (RUTKUNAS et al., 2011). A escolha do componente protético depende da retenção necessária, morfologia, anatomia, mucosa, função oral e aceitação do paciente (EVTIMOVSKA et al., 2009). O sistema de retenção protética utilizado no presente relato de caso foi o o'ring por se tratar de implante único. Tal sistema transfere menos estresse para o implante e produz menos força-momento do que o sistema de barra/clipe. Tal sistema visa reduzir a transmissão do estresse ao tecido ósseo periimplantar e minimizar o movimento da prótese (FAJARDO; ZINGARO; MONTI, 2014). Manju e Sreelal (2013) também sugerem que os componentes do o'ring possam fornecer um sistema adequado para reduzir o estresse do implante e promover a estabilidade da prótese. $\mathrm{O}$ idoso reabilitado neste relato mostrou-se extremamente satisfeito com a retenção da prótese, com acompanhamento clínico de um ano após a confecção da prótes, não relatando traumatismos ou tensões na fibromucosa que reveste o rebordo residual inferior a partir da utilização de um único encaixe do tipo óring, achados que vêm ao encontro da literatura consultada.

Manju e Sreelal (2013) afirmaram que a manutenção diária de sobredentaduras retidas pelo sistema o'ring é mais fácil do que a das overdentures retidas pelo sistema barra-clipe. Considerando as vantagens da retenção dos componen- 
tes do o-ring, especialmente na higiene, custo-efetividade e facilidade de uso, justifica-se a seleção desse sistema em pacientes geriátricos como o paciente do caso em questão, cuja higiene da prótese foi relatada como sendo simples e efetiva.

Observou-se neste estudo que o tempo cirúrgico e o custo de um implante e prótese final é menor do que overdentures retidas por 2 implantes. Embora a sobredentadura mandibular retida por dois implantes na região interforaminal seja o padrão-ouro para a reabilitação de mandíbulas edêntulas (TAKANASHI $e t$ al., 2004, THOMASON et al., 2003), as evidências mostram que a instalação de um único implante e overdenture leva a melhores resultados em comparação com a prótese total inferior convencional, como o aumento da satisfação do paciente e qualidade de vida relacionada à saúde bucal. No entanto, não há evidências confiáveis sobre o número ideal de implantes necessários para reter uma sobredentadura mandibular. A redução do número de implantes para um único implante para retenção de prótese dentária foi proposta como uma alternativa menos invasiva e onerosa comparada a outros tipos de overdentures (CORDIOLI; MAJZOUB; CASTAGNA, 1997; HARDER et al., 2011; NOGUEIRA; ESFANDIARI; LELES, 2016).

Os precursores da técnica de implante único na região mediana da mandíbula foram Cordioli; Majzoub; Castagna (1997), na Itália, em 21 pacientes com idade média de 74,2 anos e com protocolo de carga convencional (reabertura após 4 meses), com o sistema de retenção pilar bola/o'ring de borracha e acompanhamento longitudinal desses indivíduos por 5 anos consecutivos. Tal estudo demonstrou taxa de sucesso dos implantes e da prótese de $100 \%$, perda óssea marginal mínima com média de $1,42 \pm 0,56 \mathrm{~mm}$ após 60 meses. Os autores concluíram que essa é uma alternativa terapêutica para pacientes idosos passando por dificuldades de desconforto e limitações funcionais da prótese total inferior convencional, inserindo-os na sociedade e minimizando as chances de isolamento e depressão.

Liddelow e Henry (2007) desenvolveram na Austrália um ensaio clínico no qual investigaram a previsibilidade do tratamento com overdentures mandibulares utilizando abordagem cirúrgica de uma fase e carga imediata de implante unitário na mandíbula com acompanhamento de 1 ano. A satisfação dos pacientes aumentou, com diferença significativa em todos os parâmetros de conforto e função em relação à prótese convencional. O protocolo relativamente simples, custo de componentes reduzido e envolvimento laboratorial menor implicam que um número maior de indivíduos desdentados possa se beneficiar de uma prótese sobre implante.

No presente estudo, foi possível evidenciar a retenção e estabilidade do aparelho protético e o aumento da satisfação relatada pelo paciente em relação à prótese total inferior convencional que o idoso utilizava anteriormente, semelhante ao relatado em outros trabalhos.

Os desfechos clínicos, a qualidade de vida relacionada à saúde oral (OHRQoL) 
e a capacidade subjetiva de mastigar alimentos duros e macios, antes e após o tratamento com próteses totais inferiores retidas por um implante foram avaliados em 11 pacientes desdentados totais (idade média de 66,7 anos) (HARDER et al., 2011). Houve uma taxa de $100 \%$ de sucesso na osseointegração dos implantes, 4 próteses precisaram de reparo devido a fratura da base na região de linha média e notou-se melhora significante no índice de satisfação (OHRQoL), bem como na capacidade mastigatória. No presente trabalho, notou-se também uma perfeita osseointegração, bem como, o paciente relatou melhora da capacidade mastigatória.

Os desfechos clínicos da overdenture mandibular retida por implante unitário foram comparatos com os desfechos de uma prótese total convencional em 13 indivíduos (idade média de 71 anos). Nenhum implante foi perdido, não houve fraturas em próteses durante os 3 meses de observação e percebeu-se melhora significativa em todos os parâmetros de satisfação e também na capacidade de mastigação. A overdenture mandibular retida por um implante é uma alternativa promissora, especialmente para pacientes com mandíbulas atróficas, doenças sistêmicas severas e dificuldades financeiras, pois apresenta custos e tempo de tratamento reduzido e satisfação comparável (CHENG et al., 2012).

Estudos que compararam a eficiência mastigatória e grau de satisfação entre 1 ou 2 implantes, observaram não haver diferenças significativas entre 1 ou 2 implantes para reter uma prótese inferior (KRONSTROM et al., 2010; KRONSTROM et al., 2014). Mesmo diante de um tempo de acompanhamento relativamente curto, a overdenture mandibular retida por um único implante na linha média é uma alternativa ao padrão de overdenture com 2 implantes em pacientes com dificuldades de adaptação da prótese convencional (WALTON; GLICK; MACENTEE, 2009). Neste relato de caso, a overdenture retida por um único implante instalado em sínfise mandibular mostrou-se mais efetiva quando comparada com a prótese total inferior convencional.

Tendo em vista os estudos anteriormente descritos, pode-se afirmar, com base no caso ora descrito, que a confecção de uma overdenture mandibular sobre implante único, respeitando o período convencional de carregamento (4 meses), foi um tratamento bem-sucedido na cavidade bucal de paciente idoso. Neste cenário, são necessários estudos adicionais com aplicação de protocolo de carga imediata comparativamente ao carregamento convencional do implante, atentando-se principalmente a aspectos como a perda óssea marginal, sobrevida do implante e manutenção da prótese. $\mathrm{O}$ acompanhamento longitudinal dos pacientes, a partir da realização de ensaios clínicos, poderá confirmar os benefícios e a viabilidade desta técnica, tanto em relação à manutenção da retenção e estabilidade da prótese quanto à sobrevida dos implantes. 


\section{Considerações finais}

Overdentures inferiores retidas por um único implante e encaixe do tipo o'ring/bola demonstram aspectos positivos em comparação ao tratamento com próteses totais convencionais. O tratamento é efetivo para indivíduos com condições específicas em que a simplicidade da técnica apresenta vantagens, sendo os procedimentos técnicos, cirúrgicos e protéticos relativamente rápidos $\mathrm{e}$ simples, com possibilidade de benefícios imediatos (retenção, estabilidade e estética do aparelho protético e satisfação do paciente) quando comparados à prótese total convencional, além de serem pouco invasivos. Por se tratar de um relato de caso clínico, o presente trabalho apresenta algumas limitações que impedem extrapolar os resultados obtidos a uma população maior. Porém, com base nas observações, o tratamento se mostra promissor e merece ser estudado.

\section{Single implant retained overdenture: A alternative for oral rehabilitation of the elderly}

\section{Abstract}

With the increase in life expectancy, there is an increase in the demand of edentulous patients for a rehabilitation that provides them with greater masticatory efficiency. Overdentures on implants using plug-in systems represent mucosal supported and implanted prosthetic modality that enables safety, stability and aesthetics, lower cost and ease of cleaning. Internationally, two implants are considered adequate to maintain an overdenture in the toothless jaws. However, installing two implants can be an invasive and costly procedure. This work aims to demonstrate that the insertion of a single implant in the central portion of the mandible can stabilize the total prosthesis and improve the quality of life and the masticatory function of oral health. The study deals with a case report of a 65-year-old male patient who reported lack of stability of the conventional lower total prosthesis, made several years ago. The proposed treatment was the installation of a single implant in the mandibular symphysis and subsequent manufacture of new total prostheses. A fixation with an $\mathrm{O}$ ring abutment and screw-activated matrix was used to maintain the lower overdenture. The clinical results were promising, with the patient reporting improvement in chewing, stability and function of the prosthesis retained by a single implant. However, for this method to be recommended for general clinical application, results from longitudinal clinical studies are necessary.

Keywords: Overdenture. Implants. Single implant. Elderly.

\section{Referências}

ABOU-AYASH, S.; VON MALTZAHN, N.; PASSIA, N.; FREITAG-WOLF, S.; REISSMANN, D. R.; LUTHARDT, R. G.; MUNDT, T.; RAEDEL, M.; RAMMELSBERG, P.; WOLFART, S.; KERN, M. Stabilizing mandibular complete dentures by a single midline implant influence on quality of life: 2 -year results from a randomized clinical trial comparing different loading protocols. Clinical Oral Investigations; Germany, v. 24 n. 2, p. 927-35, 2020. Doi:10.1007/s00784019-02969-y.

BRYANT, S. R.; WALTON, J. N.; MACENTEE, M. I. A 5-year randomized trial to compare 1 or 2 implants for implant overdentures. Journal of Dental Research, 
Alexandria, v. 94, n. 1, p. 36-43, 2015. Doi: $10.1177 / 0022034514554224$.

CANALLATOS, J. E.; HOBBS, G. R.; BRYINGTON, M. S; DYE, B. D. The effect of implant prosthesis complications on patient satisfaction. The Journal of Prosthetic Dentistry; United States, v. 123, n. 2, p. 269-276, 2020. Doi: 10.1016/j.prosdent.2018.11.015.

CARLSSON, G. E.; OMAR, R. The future of complete dentures in oral rehabilitation. A critical review. Journal of Oral Rehabilitation, Alabama, v. 37, n. 2, p. 143-156, 2010. Doi: $10.1111 / \mathrm{j} .1365-2842.2009 .02039 . \mathrm{x}$

CHENG, T.; MA, L.; LIU, X. L.; SUN, G. F.; HE, X. J.; HUO, J. Y.; WANG; Y. N. Use of a single implant to retain mandibular overdenture: A preliminary clinical trial of 13 cases. Journal of Dental Sciences, London, v. 7, n. 3, p. 261-266, 2012. Doi: 10.1016/j. jds.2012.02.001

CORDIOLI, G.; MAJZOUB, Z.; CASTAGNA, S. Mandibular overdentures anchored to single implants: A five-year prospective study. The Journal of Prosthetic Dentistry, Iwoa, v. 78, n. 2, p. 159-165, 1997. Doi: 10.1016/S0022-3913(97)70120-3

CRITCHLOW, S. B.; ELLIS, J. S. Prognostic indicators for conventional complete denture therapy: a review of the literature. Journal of Dentistry, Singapore, v. 38, n. 1, p. 2-9, 2010. Doi:10.1016/j.jdent.2009.08.004

EVTIMOVSKA, E.; MASRI, R.; DRISCOLL, C. F.; ROMBERG, E. The change in retentive values of locator attachments and hader clips over time. Journal of Prosthodontics, Kansas City, v. 18, n. 6, p. 479-483, 2009. Doi: 10.1111/j.1532-849X.2009.00474.x

FAJARDO, R. S.; ZINGARO, R. L.; MONTI, L. M. System retained O'ring and bar-clip overdenture designs. Archives of Health Investigation, Araçatuba, v. 3, n. 1, p. 77-86, 2014. Disponível em: <http://hdl.handle. net/11449/133258>.
GROVER, M.; VAIDYANATHAN, A. K.; VEERAVALLI, P. T. OHRQoL, masticatory performance and crestal bone loss with single-implant, magnet-retained mandibular overdentures with conventional and shortened dental arch. Clinical Oral Implants Research, Copenhagen, v. 25, n. 5, p. 580586, 2014. Doi: 10.1111/clr.12111.

HARDER, S.; WOLFART, S.; EGERT, C.; KERN, M. Three-year clinical outcome of single implant-retained mandibular overdentures--results of preliminary prospective study. Journal of Dentistry, London, v. 39 , n. 10 , p. $656-661,2011$. doi: 10.1016/j. jdent.2011.07.007.

HUGO, F. N.; HILGERT, J. B.; DE SOUSA, M. D. L. R.; DA SILVA, D. D.; PUCCA, G. A. JR. Correlates of partial tooth loss and edentulism in the Brazilian elderly. Community Dentistry and Oral Epidemiology, Sydney, v. 35, n. 3, p. 224-232, 2007. Doi: 10.1111/j.0301-5661.2007.00346.x.

HYLAND, R.; ELLIS, J.; THOMASON, M.; EL-FEKY, A.; MOYNIHAN, P. A qualitative study on patient perspectives of how conventional and implant-supported dentures affect eating. Journal of Dentistry, London, v. 37 , n. 9 , p. $718-723,2009$. Doi: $10.1016 /$ j. jdent.2009.05.028.

IBGE (Instituto Brasileiro de Geografia e Estatística). Agência IBGE Notícias. PNAD Contínua: Número de idosos cresce $18 \%$ em 5 anos e ultrapassa 30 milhões em 2017. Disponível em: <https://agenciadenoticias.ibge. gov.br/agencia-noticias/2012-agencia-de-noticias/noticias/20980-numero-de-idosos-cresce-18-em-5-anos-e-ultrapassa-30-milhoes-em-2017> Acesso em: 04/08/2019.

ISMAIL, H. A.; MAHROUS, A. I.; BANASR, F. H.; SOLIMAN, T. A.; BARAKA, Y. Two Years Retrospective Evaluation of Overdenture Retained by Symphyseal Single Implant Using Two Types of Attachments. Journal of International Oral Health, India, v.7, n.6, p. 4-8, 2015. 
KLEMETTI, E. Is there a certain number of implants needed to retain an overdenture? Journal of Oral Rehabilitation, Alabama, v. 35, n. 1, p. 80-84, 2008. Doi: 10.1111/j.13652842.2007.01825.x.

KRONSTROM, M.; DAVIS, B.; LONEY, R.; GERROW, J.; HOLLENDER, L. A prospective randomized study on the immediate loading of mandibular overdentures supported by one or two implants: a 12 months follow-up report. International Journal of Oral and Maxillofacial Implants, London, v. 25, n. 1, p. 181-188, 2010.

KRONSTROM, M.; DAVIS, B.; LONEY, R.; GERROW, J.; HOLLENDER, L. A Prospective Randomized Study on the Immediate Loading of Mandibular Overdentures Supported by One or Two Implants; A 3 Years Follow-Up Report. Clinical Implant Dentistry and Related Research, New York, v. 16, n. 3, p. 323-329, 2014. Doi: 10.1111/cid.12006.

LAHOTI, K.; PATHRABE, A.; GADE, J. Stress analysis at bone-implant interface of single- and two-implant-retained mandibular overdenture using three-dimensional finite element analysis. Indian Journal Dental Research, New Delhi, v. 27, n. 6, p. 597601, 2016. Doi: 10.4103/0970-9290.199587

LIDDELOW, G. J.; HENRY, P. J. A prospective study of immediately loaded single implant retained mandibular overdentures: preliminary one-year results. Journal of Prosthetic Dentistry, Iwoa, v. 97, n. 6, p. 126-137, 2007. Doi: 10.1016/S00223913(07)60016-X

MAHOORKAR, S.; BHAT, S.; KANT, R. Single implant supported mandibular overdenture: A literature review. Journal of Indian Prosthodontic Society, India, v.16, n.1, p.7582, 2016. Doi: 10.4103/0972-4052.164881

MANJU, V.; SREELAL, T. Mandibular implant-supported overdenture: an in vitro comparison of ball, bar, and magnetic attachments. Journal of Oral Implantology, Chicago, v. 39, n. 3, p. 302-307, 2013. Doi: 10.1563/AAID-JOI-D-11-00050
MUSACCHIO, E.; PERISSINOTTO, E.; BINOTTO, P.; SARTORI, L.; SILVA-NETTO, F.; ZAMBON, S., MANZATO, E.; CORTI, M. C.; BAGGIO, G.; CREPALDI, G. Tooth loss in the elderly and its association with nutritional status, socio-economic and lifestyle factors. Acta Odontologica Scandinavica, Copenhagen, v. 65, n. 2, p. 78-86, 2007. Doi: $10.1080 / 00016350601058069$.

NICO, L. S.; ANDRADE, S. S. C. A.; MALTA, D. C.; PUCCA JÚNIOR, G. A.; PERES, M. A. Saúde Bucal autorreferida da população adulta brasileira: resultados da Pesquisa Nacional de Saúde 2013. Ciência \& Saúde Coletiva, Rio de Janeiro, v. 21, n. 2, p. 389-398, 2016. Doi: 10.1590/141381232015212.25942015

NISCHAL, K.; CHOWDHARY, R. Early Loaded Single Implant Reinforced Mandibular Overdenture. Case Reports in Dentistry, London, v. 4, n. 21, p. 37-53, 2016. Doi: $10.1155 / 2016 / 4213753$

NOGUEIRA, T. E.; ESFANDIARI, S.; LELES, C. R. Cost-effectiveness analysis of the single-implant mandibular overdenture versus conventional complete denture: study protocol for a randomized controlled trial. Trials, London, v. 17, n.1, p. 533, 2016. Doi: 10.1186/s13063-016-1646-0

NOGUEIRA, T. E.; AGUIAR, F. M. O.; DE BARCELOS, B. A.; LELES, C. R. A 2-year prospective study of single-implant mandibular overdentures: Patient-reported outcomes and prosthodontic events. Clinical Oral Implants Research, Copenhagen, v. 29 , n. 6 , p. 541-550, 2018. Doi: 10.1111/ clr. 13151

PASSIA, N.; WOLFART, S.; KERN, M. Sixyear clinical outcome of single implantretained mandibular overdentures- a pilot study. Clinical Oral Implants Research, Copenhagen, v. 26, n. 10, p. 1191-1194, 2015. Doi: $10.1111 /$ clr. 12427 . 
PATIL, P. G.; SEOW, L. L. Crestal bonelevel changes and patient satisfaction with mandibular overdentures retained by one or two implants with immediate loading protocols: A randomized controlled clinical study. Journal of Prosthetic Dentistry, London, v. 123, n. 5, p. 710-716, 2020. Doi: 10.1016/j. prosdent.2019.07.015

RUTKUNAS, V.; MIZUTANI, H.; TAKAHASHI, H.; IWASAKI, N. Wear simulation effects on overdenture study attachments. Dental Materials Journal, Michigan, v. 30, n. 6, p. 845-853, 2011. Doi: 10.4012/ dmj.2011-057.

SCHUSTER, A. J.; MACHADO, R. M. M.; BIELEMANN, A. M.; NASCIMENTO, G. G.; PINTO, L. R.; DELBEL CURY, A. A.; FAOT, F. Short-term quality of life change perceived by patients after transition to mandibular overdenture. Brazilian Oral Research, São Paulo, v. 27, n. 31, p. e5, 2017. Doi: 10.1590/1807-3107BOR-2017. vol31.0005

SMITH, B.; BAYSAN, A.; FENLON, M. Association between oral health impact profile and general health scores for patients seeking dental implants. Journal of Dentistry, Singapore, v. 37, n. 5, p. 357-359, 2009. Doi: 10.1016/j.jdent.2009.01.004

TAVAKOLIZADEH, S.; VAFAEE, F.; KHOSHHAL, M.; EBRAHIMZADEH, Z. Comparison of marginal bone loss and patient satisfaction in single and doubleimplant assisted mandibular overdenture by immediate loading. The Journal of Advanced Prosthodontics, v. 7, n. 3, p. 191-198, 2015. Doi: 10.4047/jap.2015.7.3.191

TAKANASHI, Y.; PENROD, J. R.; LUND, J. P.; FEINE, J.S. A cost comparison of mandibular two-implant overdenture and conventional denture treatment. The International Journal of Prosthodontics, Chicago, v. 17, n. 2, p. 181-186, 2004. Doi: 10.1016/j. prosdent.2004.06.016
THOMASON, J. M.; LUND, J. P.; CHEHADE, A.; FEINE, J. S. Patient satisfaction with mandibular implant overdentures and conventional dentures 6 months after delivery. The International Journal of Prosthodontics, Chicago, v. 16, n. 5, p. 467-473, 2003. Doi: 10.1016/j.prosdent.2003.12.011

WALTON, J. N.; GLICK, N.; MACENTEE, M. I. A randomized clinical trial comparing patient satisfaction and prosthetic outcomes with mandibular overdentures retained by one or two implants. The International Journal of Prosthodontics, Chicago, v. 22, n. 4, p. 331-339, 2009.

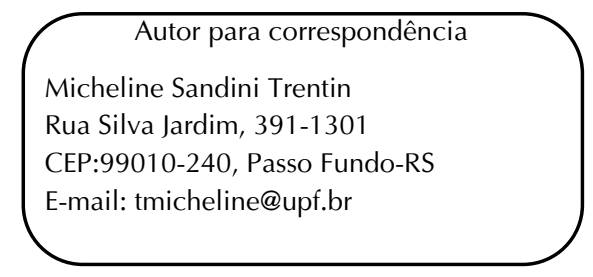

